\title{
Energy Efficiency Mechanisms in Wireless Sensor Networks: A Survey
}

\author{
Anandbabu \\ Kalpataru Institute of \\ Technology \\ Tipur, Karantaka, India
}

\author{
Siddaraju \\ Dr Ambedkar Institute of \\ Technology \\ Bengaluru, Karnataka, India
}

\author{
Guru R. \\ Sjce, Mysore
}

\begin{abstract}
Wireless Sensor Network (WSN) is one of the emerging research domains which have posed numerous unique challenges to researchers. The low cost MEMS and low processing capability with ultra low power consumption and low range wireless communication makes it lucrative for being exploited in abundance in future. The inbuilt limitation of energy in the sensor nodes which demanded every functionality of WSNs to be energy efficient. In this survey, an attempt is made to study the energy efficient approaches in WSNs. Classification of energy efficient approaches in WSNs is proposed and It also overviews ongoing research approaches, and provides discussion and provision of some design issues and requirements for building energy efficient mechanisms for WSNs.
\end{abstract}

\section{Keywords}

WSNs, Energy Efficiency, Security, Routing, Coverage, Localization

\section{INTRODUCTION}

Wireless Sensor Networks (WSNs) have emerged as a new computing paradigm within the past few years. The small size of sensors can be deployed physically, where each can sense information from the phenomena and they can communicate each other or sink node. Interested users can extract useful information about the environment from the WSNs[1][2][3]

Generally, the WSNs consist of large number of sensor nodes, which are densely deployed in an area. The sensor nodes are application specific. As they are battery operated, the energy level has to be considered for all sorts of operations. Each sensor is self configurable by itself to connect to neighboring sensor and establish network

The sensor nodes cooperate on the detecting task and use a wireless link for communication. The wireless network has been formed among sensor nodes. The connection between others network has also established. Using the wireless communication yield the WSNs can be conveniently constructed since no infrastructure is requested. Here, source nodes detect the event then they transmit information to sink nodes. After receiving information packet, the sink nodes store them in their memory or process them. The sink nodes might be sensor nodes in the WSNs, PDA or internet gateway where user uses for transmitting his data request. The data transmission from source node to sink node cannot be directly done in large WSNs due to limitation of wireless communication range; thus the most of data transmissions are in multi-hops form.

The sensor node is consisted of five modules - Controller (a CPU is used for processing, data collection and data path searching), Memory module (for storing the sensed information as well as other data), Communication Devices
(Transceiver for transmission of information), Sensor and Actuator modules (for sensing the data and finding the location of system) and power supply (energy source of sensor node, mainly battery power). The energy depletion in the sensor node due to sensing, in-network processing/logging, receiving data from source/relay (along with overhearing), transmitting sensed/relayed data (including over-emitting, collision, channel polling and control packet overhead), startup transient energy (may increase due to frequent switching), idle state (including idle-listening) and sleep state[4][5][6].

A sensor node would drain its battery faster in the absence of energy efficient techniques. This fact triggered researchers to design protocols and mechanisms to reduce energy utilization in the WSNs. Many researchers proposed study on energy efficiency techniques in MAC layer, routing layer, transport and application layer separately.

In this survey, it is attempted to present different energy saving mechanisms in WSNs protocol stack across all the layers and present systematic classification of energy saving mechanisms for WSNs. This paper also presents open issues in energy efficiency techniques for WSNs.

The reminder of this paper is organized as follows. Section II discusses Background and Energy Efficiency in WSNs. The aim of Section III is to present systematic classification of energy efficiency techniques for WSNS. Section IV discusses Open Issues and Challenges, and We conclude in Section V.

\section{BACKGROUND AND ENERGY EFFICIENCY IN WSNS}

Most of the node energy is spent in the communications compared to data sensing and processing. The maximum energy is spent in the communication process (transmit $(14.88 \mathrm{~mW}) \&$ receive mode $(12.50 \mathrm{~mW}))$, then, next to it is the energy spent in idle mode $(12.36 \mathrm{~mW})$ [7]. Although, a minimum amount of energy is spent in sensing, sleeping \& processing mode, still they consume some energy. These processes need to be energy-efficient.

To prolong the operational network lifetime of WSNs optimum use of limited available power source is needed. In WSNs, the energy expense can be minimized in all the layers of the protocol stack. In Physical layer, the node energy can be saved through reducing the data size, effective data rate and efficient energy model. In MAC layer, designing energy efficient MAC duty cycle mechanisms and packet scheduling. Energy efficient routing protocol can be designed to reduce the energy consumption in the network layer. In transport layer, effective congestion control, congestion avoidance and load sharing mechanisms contribute in enhancing the network lifetime

Data placement and asynchronous multicasting techniques 
attempts to reduce energy expense at application layer. Energy spent in sensor nodes is much in communication compared to sensing and data processing.

\section{CLASSIFICATION OF ENERGY EFFICIENCY TECHNIQUES FOR WSNS}

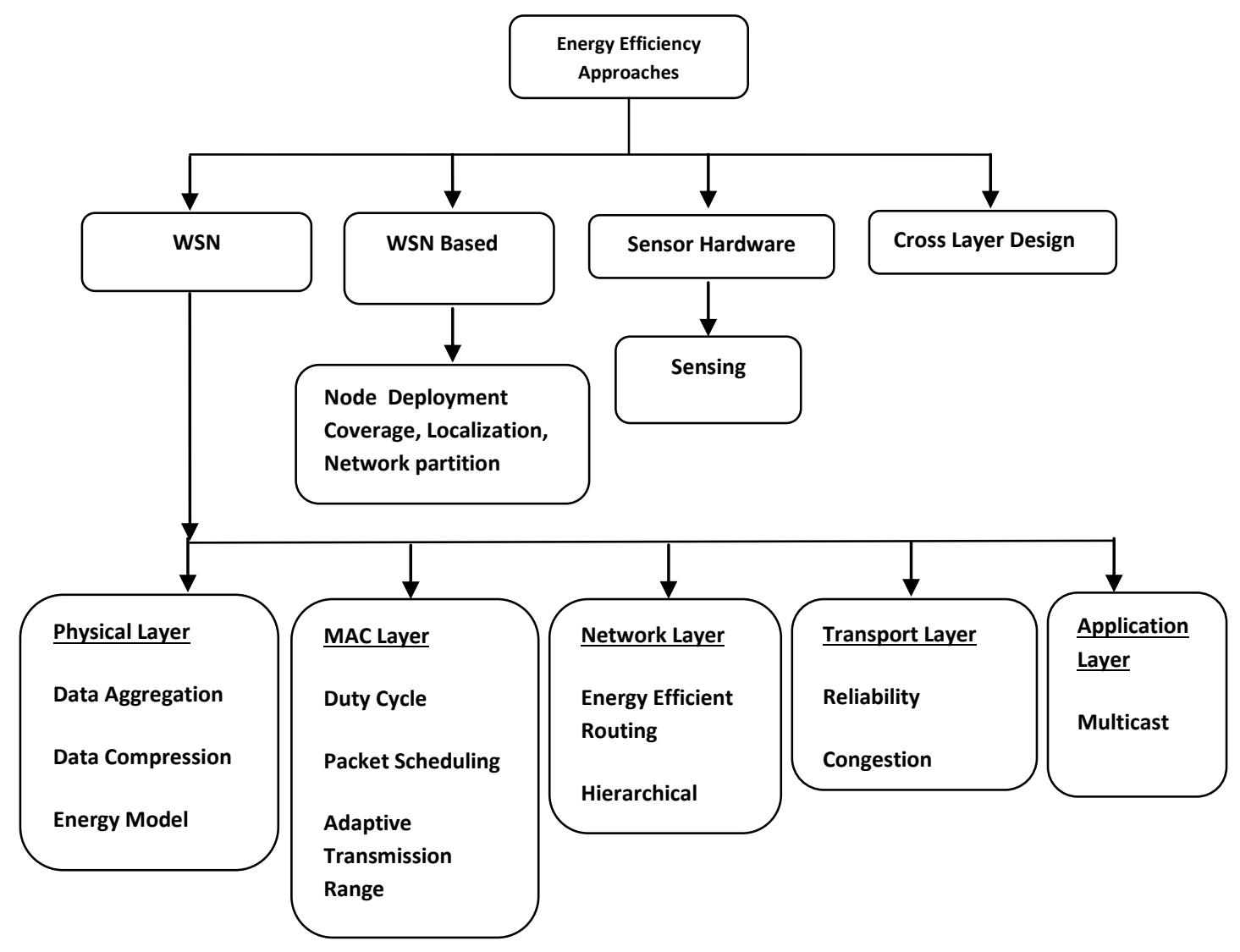

Fig. 1 Classification of Energy efficiency mechanisms in WSNs

The classification of Energy efficiency mechanisms in WSNs can be classified as WSN Protocol Stack, WSN based Techniques, Sensor Hardware and Cross layer design mechanisms as shown in the Fig. 1.

\subsection{WSN Protocol Stack}

The energy efficient techniques under WSN Protocol Stack can further classified as physical layer, mac layer, network layer, transport layer and application layer. The energy conserving technique in each of the WSN Protocol Stack is discussed[8].

\subsubsection{Physical Layer}

The sensor output is usually an analog signal. After signal conditioning (amplification, filtering) and digitization, data are processed locally in the node. The sensor senses the physical data from the phenomena and usually it is in analog in nature. The sensed data need to be amplified and modulated in order to minimize the loss of information. Each of these processes involves an electronic circuit that consumes a considerable level of energy[8][9]. Modulation, transmission $\&$ receiving techniques are importance of physical layer. Data Rate, Data reduction, Energy Model \& Sampling Workload Allocation energy conservation techniques in physical layer of WSNs.
Holland et al[10] proposed a method of finding the optimum physical layer parameters to minimize energy dissipation in a multi-hop wireless sensor network. A metric that specifies the energy per successfully received bit (ESB). Which is function of three physical layer parameters: hop distance, $d$, transmit energy, Es,TX, and the modulation scheme. ESB also depends on the channel model. Et al proposed specific channel model and a constraint on any two of the three physical layer parameters. It minimize energy dissipation and hence optimize the performance of the network.

Eugene et al [11] proposed the design of physical layer aware protocols, algorithms, and applications that minimize energy consumption of the system and individual nodes. The authors also proposed algorithms that take advantage of these adjustable hooks and knobs to minimize energy.

\subsubsection{Mac Layer}

The MAC layer has to be responsible for reliability, energy efficiency, high throughput \& low access delay to optimally utilize the energy-limited resources of sensor nodes. Maximum amount of energy wasted in MAC protocol operations like collision, overhearing, control packet overhead and interference. To minimize the energy expenditure at WSNs energy efficient MAC techniques like duty cycling, 
packet scheduling adaptive transmission range, and adaptive transmission period[12][13][14]. A duty cycling in MAC layer involves the sensor node to sleep/wake up mechanisms to conserve energy. Sleep/wake up mechanisms involves in putting the radio transceiver in the (low-power) sleep mode whenever communication is not required. Ideally, the radio should be switched off as soon as there is no more data to send/receive, and should be resumed as soon as a new data packet becomes ready. The mechanism which makes the sensor nodes to alternate between active and sleep periods depending on network activity can be referred as duty cycling.

Kein et al [15] proposed the low overhead MAC protocol (LO-MAC), a new duty cycling MAC protocol, which reduces the control overhead on data transmission. LO-MAC uses carrier sensing techniques to bypass the long Listening period problem, which occurs very frequently under low traffic load environments. After the Sync period, a short period called the carrier sensing period is introduced. In this period, LO-MAC employees the carrier sensing technique to inform the node traffic status and then decide to turn their radios off or keep them on to involve in a possible data transmission. LO-MAC is also able to transmit data packets over multiple hops in a single cycle and thoroughly use the broadcast nature to minimize the control overhead.

\subsubsection{Network Layer}

Routing is the process which finds the path between the source to destination while initiating data communication in the network. Routing is much more important than any other networks compared to WSNs.

Table 1. Radio Characteristics, Classical model

\begin{tabular}{|l|l|}
\hline \multicolumn{1}{|c|}{ Radio mode } & $\begin{array}{l}\text { Energy } \\
\text { Consumption }\end{array}$ \\
\hline $\begin{array}{l}\text { Transmitter Electronics (EelecTx) } \\
\text { Receiver Electronics }(E-\text { elecRx }) \\
(\text { Eelec=EelecRx }=\text { EelecTx })\end{array}$ & $50 \mathrm{~nJ} / \mathrm{bit}$ \\
\hline Transmit Amplifier (Eamp) & $100 \mathrm{pJ} / \mathrm{bit} / \mathrm{m}^{2}$ \\
\hline Idle (Eidle) & $40 \mathrm{~nJ} / \mathrm{bit}$ \\
\hline Sleep & 0 \\
\hline
\end{tabular}

The energy spent in communication (transmission and reception) is much higher than Idle and sleep state of the sensor node. It shows that energy saving in data communication is needed in the WSNs. The sensed data communicating to the sink node involves transmission from the source and intermediate nodes needs to receive and transmit the data to the next neighboring nodes. Energy efficient routing can be done through hierarchical Routing, flat routing, multipath routing, geographical routing etc.

Rasheed et al [16] stated the efficient use of energy source in a sensor node is most desirable criteria for prolong the life time of wireless sensor network. The authors in [4] proposed a two layer hierarchical routing protocol called Cluster Based Hierarchical Routing Protocol (CBHRP). They introduced a new concept called head-set, consists of one active cluster head and some other associate cluster heads within a cluster. The head-set members are responsible for control and management of the network. Results show that this protocol reduces energy consumption quite significantly and prolongs the life time of sensor network as compared to LEACH. An effective routing protocol is designed and tested it performances to overcome some present limitation of WSN. Authors stated that introducing head-set concepts instead of only one cluster head within a cluster, their results show the better performance than that of LEACH in context of energy consumption, frame transmission and the life time of the sensor network.

Heinzelman et al [17] proposed LEACH Protocol. It is a kind of cluster-based routing protocols, which includes distributed cluster formation. LEACH randomly selects a few sensor nodes as cluster heads $(\mathrm{CHs})$ and rotates this role to evenly distribute the energy load among the sensors in the network. The idea is to form clusters of the sensor nodes based on the received signal strength and use local cluster heads as routers to the sink. In $\mathrm{LEACH}$, the $\mathrm{CH}$ nodes compress data arriving from nodes that belong to the respective cluster, and send an aggregated packet to the BS in order to reduce the amount of information that must be transmitted to the BS. LEACH uses a Time division multiple access (TDMA) and code-division multiple access (CDMA) MAC to reduce inter-cluster and intra-cluster collisions. All the data processing such as data fusion and aggregation are local to the cluster. The operation of LEACH is done into two phases, the setup phase and the steady state phase. In setup phase the clusters are organized and $\mathrm{CHs}$ are selected. Cluster heads change randomly over time in order to balance the energy dissipation of nodes. This decision is made by the node choosing a random number between 0 and 1 . The node becomes a cluster head for the current round if the number is less than the following threshold value $\mathrm{T}(\mathrm{n})$.

$$
\mathrm{T}(\mathrm{n})=\left\{\begin{array}{c}
\frac{P}{1-P\left(\operatorname{rmod}\left(\frac{1}{P}\right)\right)}, \text { if } n \in G, \\
0, \text { otherwise }
\end{array}\right.
$$

Where, $\mathrm{G}$ is the set of nodes that are involved in the $\mathrm{CH}$ election. In the steady state phase, the actual data transfer to the BS takes place. The duration of the steady state phase is longer than the duration of the setup phase in order to minimize overhead. During the steady state phase, the sensor nodes can begin sensing and transmitting data to the $\mathrm{CHs}$. The $\mathrm{CH}$ node, after receiving all the data, aggregates it before sending it to the BS. After a certain time, which is determined a priori, the network goes back into the setup phase again and enters another round of selecting new CHs. Each cluster communicates using different CDMA codes to reduce interference from nodes belonging to other clusters.

\subsubsection{Transport Layer}

Out of sight problem is also common in WSNs as in wireless networks. When multiple nodes want to transmit data through the same channel at a time or when the routing node fails to forward the received data to the next routing nodes congestion occurs. Congestion and data loss occurs at nodes which are nearer to the sink nodes [18][19].

Energy saving can be achieved in transport layer of WSNs through energy aware congestion avoidance, energy efficient load sharing and energy efficient reliable mechanisms between end to end communication in WSNs.

Awais et al[20] proposed congestion control mechanism which considers node level congestion (NLC) and link level congestion (LLC). The NLC is caused by the buffer overflow whereas LLC is caused by large data being pumped into the channel by various neighboring nodes at the same time. It is designed based on statistical time division multiple access (STDMA) that improves performance in both NLC and LLC 
and ultimately results in energy efficiency of the network. With the help of STDMA, each MSN shares its statistical information in Hello Packet with cluster head $(\mathrm{CH})$. This Hello Packet contains unique ID, battery information, and location information in the cluster. The $\mathrm{CH}$ then uses MSN feedback and uses a modified TDMA technique of time slots allocation to its MSNs in specific cluster.

\subsubsection{Application Layer}

Energy saving in the application layer is achieved through application service which aims at energy conservation, by caching mutable data obtained from data-retrieval at locations that minimize the sum of request and update traffic \& asynchronously multicasting updates from sensors to observers reduces the total number of packet transmissions in the network.

Bhattacharya et al[21] developed a distributed framework that improves power conservation by application-layer sensor data caching and asynchronous update multicast. The goal of the framework is to reduce the total power expended on the primary network function; namely, data collection and delivery.

\subsection{WSN based Techniques}

The energy efficiency is achieved through effective node deployment in the phenomena, one coverage mechanisms in the network, effective node location in the phenomena and optimal network partition techniques.

\subsubsection{Energy Efficient Deployment Scheme in WSNS}

The initial deployment is hard to control using such deployment mechanisms. The optimal deployment of nodes also adds to the lifetime of the network, along with determination of deployment cost, coverage, connectivity, etc. A good deployment is important to achieve load balance and prolong the network lifetime [22][23].

Authors in [23] proposed a distributed deployment scheme (DDS) for homogeneous distribution of Mobile sensor Nodes (MSNs) within the candidate region. The candidate region is logically divide into concentric layers centered at it and each layer is separated by half of rc as shown in the Fig 2. desired locations for placement of MSNs is computed by Base station.

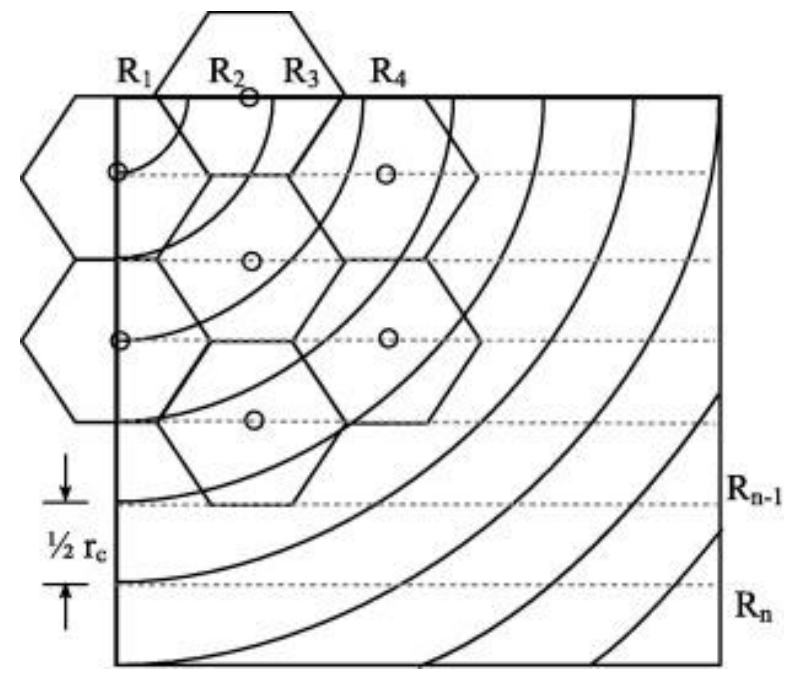

Fig 2. The Candidate Region

Desired locations are computed by dividing the candidate region into regular hexagons (with side $=r_{s}$ ) and center of these hexagons constitutes desired locations. The relation between $r_{s}$ and $r_{c}$ is given by Eq. (3)

$$
r_{e}=r_{p} * \sqrt{3}
$$

BS invites randomly spread MSNs layer after another (starting from inside) to occupy the desired locations, thereby getting connected. If any layer doesn't contain MSNs then BS waits for certain time $t(t \geqslant$ maximum time taken by MSN in current layer to move up to last occupied layer) and invites the MSNs in the next layer to move toward connected nodes to get connected. The process is repeated until all the nodes are placed on desired locations. DDS shows the multi-path connectivity, with minimum overlap and achieves 100\% coverage with limited MSN movement, but the scheme is not scalable as MSNs are deployed in linear fashion (layer after another) due to which Deployment time increases with the area. Moreover MSNs have mobility restricted to plain surfaces which leave the system incompetent for real time scenarios.

\subsubsection{Energy Efficient Coverage approaches in WSNS}

The coverage issues in WSNs can be viewed as a major in QoS and performance of WSNs. Coverage issues may be categorized as area coverage, target coverage and barrier coverage [24-27]. The main objective of the area coverage in WSNs is to cover a region (the collection of all space points within the sensor field), and each point of the region need to be monitored. Point coverage is to cover a set of point (target) with known location that need to be monitored. The point coverage scheme focuses on determining sensor nodes' exact positions, which guarantee efficient coverage application for a limited number of immobile points (targets).

Target coverage in WSNs targets number of known locations that needs to be continuously observed (covered) and a large number of sensors closely deployed to the target. Barrier coverage is to detect movement across a barrier of sensors [28]. The coverage issues improve minimization of power utilization of WSNs and WSNs lifetime.

\subsubsection{Energy Efficient Localization in WSNs}

Localization is a process of identifying the location of a sensor node in WSNs without using global positioning system (GPS). Localization in WSNs is basically categorized as target localization and self node localization. Source node location is estimated based on energy, angle of arrival (AOA), time difference of arrival (TDOA) [29-33].

[34] et al proposed a mechanism to obtain the appropriate number of active sensors to locate an object and reduces energy consumption in localization. Sensor nodes are triggered by the opportunistic wakeup probability sent from the user. As the number of active sensors in the sensing range of the user is decreased, and energy consumed is also reduced.

Opportunistic wakeup probability is evaluated using Trilateration localization algorithm, in two-dimensional space, it need at least three reference nodes' location information to locate an object. It assume that the nodes are independent, when the number of in-sensors $3 \geq n$, the probability of successfully locating a User obeys the binomial probability $\mathrm{B}(\mathrm{n}, \mathrm{p})$, where $\mathrm{n}$ represents the number of sensor nodes in the sensing range of the mobile object, and $\mathrm{p}$ represents the wakeup probability of sensor node. The probability of accurately locating a moving object can be obtained from (1).

$P(K \geq 3)=1-P(K==0)-P(K-1)-P(K=2)$ 
$P(K \geq 3)=1-C_{n}^{1} p(1-p)^{n-1}-C_{n}^{2} p(1-p)^{n-2}-(1-p)^{n}(2)$

where $P$ represents the probability of accurately locating the mobile object, and $\mathrm{K}$ represents the number of sensor nodes to wake up. According to (2), it is a quantitative relation between the number of in-sensors and the probability of accurately locating a User, under certain preconditions of each sensor's wakeup probability. Sensor nodes are sleeping which are not triggered by opportunistic wakeup probability. It avoid Will not participate in the communication and contributes in power consumption.

\subsubsection{Energy Efficient Security in WSNs}

WSNs are resources constrained in battery power, processing power, memory and communication range. The sensed data is send to base station in multiple hops. As there are no centralized mechanisms to monitor data communication, data communication is highly vulnerable. There is need of security measures in WSNs. High computational and communication overhead by the security mechanisms on the resource limited sensors are limiting the lifetime of the sensor nodes in the network. Self organizing and multihop communication in WSNs prone to network routing attacks such as spoofed routing information, selective packet forwarding, sybil attack, sinkhole attack, wormholes attack, hello flood attacks, and acknowledgment spoofing[36-40]. Designing a secure mechanism in data routing which defends against the routing attacks and proves its energy efficiency for WSNs is need to be addressed by the researchers.

\section{OPEN ISSUES AND CHALLENGES}

WSNs are inbuilt with number of challenges including limited node energy, limited processing capacity, limited memory, smaller transmission range, fewer sink node than sensor nodes, different kinds of sensors, etc. Along with these challenges, WSNs pose many issues to researchers to address. To improve the performance of WSNs and reduce the usage of energy and increase the lifetime of the WSNs is the common interest. The protocol stack of WSNs has issues[35].

Physical Layer is responsible for raw data transmission medium issues. It covers different kinds of sensors and connectivity, wireless networks general issues like path loss, reflection, absorption and scattering loss, interference, modulation techniques, signal quality and strength are also the major issues in WSNs.

Data link layer Data link layer's major responsibility is to ensure interoperability amongst communication between nodes. This layer deals error detection and correction, flow control, multiplexing for WSNs.

Network layer is responsible for selecting the Optimized path for the sensed data routing to sink node. It sends the data to sink node in multiple hops. Designing energy efficient routing protocols for WSNs is one of the major issues since; the energy cost for communication is higher than the processing cost. Secure data communication in the data routing is a also need to be addressed.

Handling end to end congestion related issues and connecting WSNs to the external network i.e., Internet. To establishing and maintain communication between WSNs and Internet and a dedicated transport layer protocols which addresses issues like congestion, end to end connectivity etc.

A novel and light weight Application Layer protocols are needed to to display and guarantee reliable data flow to lower layers. This layer is responsible for data collection, management and processing of the data by using the application software to obtain reliable data transmission.

Network Management in WSNs becomes important in order to keep the WSNs and application work properly. Until now, there is no optimized or considerable network management solution for WSN. Recently it is getting momentum from the research community to design Management of WSNs. It has already presented a set of significant management challenges [36][37]. The operation of a WSN is greatly affected by different inter-related factors such as network traffic flows, network topologies, and communication protocols. Specific network management architecture needs to address considering WSNs challenges and characteristics.

\section{CONCLUSION}

As a new research area, wireless sensor network has promised its usage in the future computing and communication environment. However, there still needs significant research work to address a set of research challenges and issues. One of the biggest challenges is the designing of any mechanisms and techniques for WSNs to be energy efficient. In this survey we proposed detailed classification of energy efficient approaches for WSNs. As it is many approaches proposed to improve energy efficiency and network still unique issues need to be addressed. It discussed some ongoing research work of energy efficient mechanisms in WSNs from different aspects is also presented Such issues in noted in challenges an open issues for WSNs is discussed. A detail energy efficient issues relating to protocol stack and major issues are discussed. Finally, it summaries needed energy efficient mechanisms which will improve energy efficiency and network lifetime in the future energy efficient techniques.

\section{REFERENCES}

[1] V. Raghunathan, C. Schurgers, Park.S, and M.B. Srivastava, "Energy-aware wireless microsensor networks," IEEE Signal Processing Magazine, Volume: 19 Issue: 2 , March 2002 Page(s): 40 -50.

[2] Jennifer Yick, Biswanath Mukherjee, Dipak Ghosal, "Wireless Sensor Network Survey", Computer Networks 52, pages 2292-2330, 2008.

[3] I. F. Akyildiz, W. Su, Y. Sankarasubramaniam, and E. Cayirci. "Wireless Sensor Networks: A Survey. Computer Networks", Vol 38, Issue 4, pp.393-422, March 2002.

[4] V. Raghunathan, C. Schurgers, Park.S, and M.B. Srivastava, "Energy-aware wireless microsensor networks," IEEE Signal Processing Magazine, Volume: 19 Issue: 2 , March 2002 Page(s): 40 -50.

[5] Shilpa Das , Benudhar Sahu "A Survey On Energy Conservation Techniques In Wireless Sensor Networks", International Journal of Advance Electrical and Electronics Engineering (IJAEEE), ISSN pp: 2278-8948, Volume-3 Issue-1, 2014.

[6] R. R. Rout, \& S. K. Ghosh, -Enhancement of lifetime using duty cycle and network coding in wireless sensor networksll, IEEE Transactions on Wireless Communications, vol. 12, no. 2, pp. 656- 667, Feb.2013.

[7] S. Lanzisera, A.M. Mehta, \& K.S. Pister, -Reducing average power in wireless sensor networks through data rate adaptation, Proceedings in IEEE International Conference on Communications ICC'09, pp. 1-6, June 2009. 
[8] Y. Yao and J. Gehrke, "The cougar approach to innetwork query processing in sensor networks", Special Interest Group on Management Of Data (SIGMOD) 2002, Vol 31, No 3, September 2002

[9] Sandra Sendra, Jaime Lloret, Miguel García and José F. Toledo Power saving and energy optimization techniques for Wireless Sensor Networks JOURNAL OF COMMUNICATIONS, Vol. 6, No. 6, SEPTEMBER 2011.

[10] M. Holland and T. Wang, "Optimizing Physical-Layer Parameters for Wireless Sensor Networks," ACM Transactions on Sensor Networks, vol. 7, no. 4, 2011.

[11] Eugene Shih, Seong-Hwan Cho, Nathan Ickes, Rex Min, "Physical Layer Driven Protocol and Algorithm Design for EnergyEfficient Wireless Sensor Networks" ACM SIGMOBILE pp. 272-286, 2001

[12] Yang Liu,Itamarelhanamy,hairong Qi “An energy efficient QOS-aware media access control protocol for wireless sensor networks":2005 in Proceedings of the IEEE International Conference on Mobile Ad hoc and Sensor Systems.

[13] Hu W, Chen Q, Corke P, O'Rourke D. An Energyefficient Rate Adaptive Media Access Protocol (RAMAC) for Long-lived Sensor Networks. Sensors (Basel, Switzerland). 2010;10(6):5548-5568. doi:10.3390/s100605548.

[14] Tz-Heng Hsu, Tai-Hoon Kim, Chao-Chun Chen, and Jyun-Sian Wu, "A Dynamic Traffic-Aware Duty Cycle Adjustment MAC Protocol for Energy Conserving in Wireless Sensor Networks," International Journal of Distributed Sensor Networks, vol. 2012, Article ID 790131, 10 pages, 2012. doi:10.1155/2012/790131.

[15] Ali Chamam, and Samuel Pierre , "On the Planning of Wireless Sensor Networks: Energy-Efficient Clustering under the Joint Routing and Coverage Constraint", in IEEE Transactions on MOBILE COMPUTING, August 2009 (vol. 8 no. 8) pp. 1077-1086.

[16] Yanwei Wu, Xiang-Yang Li, YunHao Liu, Wei Lou, "Energy-Efficient Wake-Up Scheduling for Data Collection and Aggregation," IEEE Transactions on Parallel and Distributed Systems, vol. 21, no. 2, pp. 275287, Feb. 2010, doi:10.1109/TPDS.2009.45

[17] Christophe J. Merlin, Wendi B. Heinzelman, Schedule Adaptation of Low-Power-Listening Protocols for Wireless Sensor Networks, IEEE Transactions on Mobile Computing, vol. 9, no. 5, pp. 672-685, May 2010 doi:10.1109/TMC.2009.153.

[18] Kien Nguyen, Yusheng Ji, and Shigeki Yamada," Low Overhead MAC Protocol for Low Data Rate Wireless Sensor Networks" International Journal of Distributed Sensor Networks Volume 2013, Article ID 217159.

[19] Md. Golam Rashed, M. Hasnat Kabir, Muhammad Sajjadur Rahim, Shaikh Enayet Ullah, "Cluster Based Hierarchical Routing Protocol For Wireless Sensor Network", in (IJCNS) International Journal of Computer and Network Security, Vol. 2, No. 5, May 2010, pp 128131.

[20] W. Heinzelman, A. Chandrakasan and H. Balakrishnan, "Energy- efficient communication protocol for wireless microsensor networks," in Proc. of the 33rd Annual
Hawaii Int' Conf. on System Sciences, Maui: IEEE Computer Society, 2000, pp. 3005-3014.

[21] S. S.Wang and Z. P. Chen, "LCM: a link-aware clustering mechanism for energy-efficient routing in wireless sensor networks,"IEEE Sensor Journal, vol. 13, no. 2,2013

[22] Saima Zafar, "A Survey of Transport Layer Protocols for Wireless Sensor Networks",International Journal of Computer Applications (0975 - 8887) Volume 33- No.1, November 2011.

[23] Ahmed Ayadi," Energy-Efficient and Reliable Transport Protocols for Wireless Sensor Networks: State-of-Art" Wireless Sensor Network, 2011, 3, pp.106-113, doi:10.4236/wsn.2011.33011.

[24] S. Bhattacharya, H. Kim, S. Prabh, \& T. Abdelzaher, "Energy-conserving data placement and asynchronous multicast in wireless sensor networks", In Proceedings of the 1st international conference on Mobile systems, applications and services, ACM, pp. 173-185, May 2003.

[25] Wen-Hwa Liao, Ssu-Chi Kuai, Mon-Shin Lin,” An Energy-Efficient Sensor Deployment Scheme for Wireless Sensor Networks Using Ant Colony Optimization Algorithm", Wireless Personal Communications, June 2015, Volume 82, Issue 4, pp 2135-2153.

[26] Vikrant Sharma, R.B. Patel, H.S. Bhadauria, and D. Prasad "Deployment schemes in wireless sensor network to achieve blanket coverage in large-scale open area: A review" Egyptian Informatics Journal(corrected Proof).

[27] D. Tian and N. D. Georganas, -A Coverage-Preserving Node Scheduling Scheme for Large Wireless Sensor Networks, Proc. of the 1st ACM Workshop on Wireless Sensor Networks and Applications, 2002.

[28] K. Kar, S. Banerjee, "Node placement for connected coverage in sensor networks" In: Proceedings of the Workshop on Modeling and Optimization in Mobile, Ad Hoc and Wireless Networks (WiOpt'03), Sophia Antipolis, France, 2003.

[29] M. Cardei, M. Thai, L. Yingshu, W. Weili , "Energyefficient target coverage in wireless sensor networks", Proceedings IEEE, Vol. 3, pp. 1976- 1984, 2005.

[30] X.-Y. Li, P.-J. Wan, and O. Frieder , "Coverage in Wireless Ad-hoc Sensor Networks",IEEE Transactions on Computers Vol. 52, pp. 753-763, 2002

[31] Megha Bisht and Sonia Chhetri, "A Survey on the Coverage of WSNs "International Journal of Advanced Research in Computer Science and Software Engineering, Volume 3, Issue 3, March 2013.

[32] L. M. Kaplan, Q. Le, and P. Molnar, "Maximum likelihood methods for bearings-only target localization," in Proceedings of IEEE International Conference on Acoustics, Speech, and Signal Processing, vol. 5, pp. 3001-3004, Salt Lake City, Utah, USA, May 2001.

[33] Y. Weng, W. Xiao, and L. Xie, "Total least squares method for robust source localization in sensor networks using TDOA measurements," International Journal of Distributed Sensor Networks, vol. 2011, Article ID 172902, 8 pages, 2011.

[34] X. Qu and L. Xie, "Source localization by TDOA with 
random sensor position errors-part I: static sensors," in Proceedings of the 15th International Conference on Information Fusion, pp. 48-53, Singapore, July 2012.

[35] X. Qu and L. Xie, "Source localization by TDOA with random sensor position errors-part II: mobile sensors," in Proceedings of the 15th International Conference on Information Fusion, pp. 54-59, Singapore, July 2012.

[36] K. C. Ho, "Bias reduction for an explicit solution of source localization using TDOA," IEEE Transactions on Signal Processing, vol. 60, no. 5, pp. 2101-2114, 2012.

[37] Feng Xia, Xue Yang, Haifeng Liu, Da Zhang and Wenhong Zhao "Energy-Efficient Opportunistic Localization with Indoor Wireless Sensor Networks" ComSIS Vol. 8, No. 4, Special Issue, October 2011

[38] Anjali Potnis1, and C S Rajeshwari "Wireless Sensor Network: Challenges, Issues and Research", Electrical \& Electronics Engineering, NITTTR, Bhopal, M.P. India

[39] Long Cheng, Chengdong Wu, Yunzhou Zhang, Hao Wu, Mengxin Li and Carsten Maple "Review Article A Survey of Localization in Wireless Sensor Network “, International Journal of Distributed Sensor Networks, Hindawi Publishing Corporation, Volume 2012, Article ID 962523, 12 pages doi:10.1155/2012/962523.

[40] A. Abduvaliyev, et al, "On the Vital areas of Intrusion Detection Systems in Wireless Sensor Networks", IEEE Communications Surveys \& Tutorials, Vol. 15, No. 3, pp. 1223-1237, 2013.

[41] N. A. Alrajeh, S. Khan, B. Shams, "Intrusion Detection Systems in Wireless Sensor Networks: A Review", International Journal of Distributed Sensor Networks, pp. $1-7,2013$.

[42] Santhosh Simon, K Paulose Jacob Energy Optimized Secure Routing Protocol for Wireless Sensor Networks International Journal of Engineering and Innovative Technology (IJEIT) Volume 3, Issue 4, October 2013.

[43] K. Lin, Ch. F. Lai, X. Liu, and X. Guan, "Energy efficiency routing with node compromised resistance in wireless sensor networks", Mob. Netw. Appl., vol. 17, pp. 75-89, 2012.

[44] K. Sharama, M. K. Ghose, "Security model for hierarchical clustered wireless sensor networks," International Journal of Computer Science and Security (IJCSS), vol. 5, No. 1, pp. 85-97, 2011.

[45] Muhammad Asim; Hala Mokhtar; Muhammad Zahid Khan ," An energy efficient management scheme for wireless sensor networks", International Journal of Critical Computer-Based Systems (IJCCBS), Vol. 6, No. 2, 2015 .

[46] Omar Said,"Performance evaluation of WSN management system for QoS guarantee" EURASIP Journal on Wireless Communications and Networking, 2015. 